\title{
TWO CHARACTERIZATIONS OF QUASI-PSEUDOMETRIZABLE BITOPOLOGICAL SPACES
}

\author{
S. ROMAGUERA \\ (Received 24 March 1982; revised 14 September 1982) \\ Communicated by G. H. Rubinstein
}

\begin{abstract}
By using pairwise relatively complete families of functions, which we define here, we obtain two characterizations of quasi-pseudometrizable bitopological spaces from which some known theorems can be derived as easy corollaries.
\end{abstract}

1980 Mathematics subject classification (Amer. Math. Soc.): primary 54 E 55; secondary 54 E 35. Keywords and phrases: bitopological spaces, quasi-pseudometrization.

\section{Introduction}

Since Kelly [4] began the study of bitopological spaces, one of the main problems has been to find necessary and sufficient conditions for quasi-pseudometrization. This problem was considered by Kelly [4], Patty [7] and Lane [5], and later by Salbany [11], who uses $\sigma-(\mathscr{P}, \mathcal{2})$ locally finite pairbases to give a sufficient condition for bitopological quasi-pseudometrization, and he obtains a bitopological version of the Nagata-Smirnov's theorem which solves a conjecture of Patty. Also, Salbany improves some quasi-pseudometrization theorems belonging to Kelly and Lane. Recently, Pareek [6] has obtained a necessary and sufficient condition for quasi-metrization of a $T_{1}$ bitopological space which generalizes a topological result of Ribeiro [9].

Here we introduce the concept of pairwise relatively complete family of functions, and we prove a necessary and sufficient condition for bitopological quasi-pesudometrization in terms of such families. We easily derive some known

(C) 1983 Australian Mathematical Society $0263-6115 / 83 \$ A 2.00+0.00$ 
theorems as corollaries, and a new characterization of quasi-pseudometric spaces by using quasi-gauges. Theorem 2.4 is a bitopological generalization of a pseudometrization theorem proved by Guthrie and Henry [2] and [3]. We also give an easy proof of Pareek's theorem referred to before.

In the following, $\mathbf{R}$ will denote the set of real numbers, and the abbreviations 1.s.c. and u.s.c. for lower and upper semicontinuous functions, respectively, are used. We use the notation of [10].

The main part of the results in this paper belongs to the author's doctoral thesis 'Sobre la pairwise normalidad y la cuasimetrización de los espacios bitopológicos' submitted on September 28th 1981 to the University of Valencia, Spain, under the direction of Dr. Manuel López Pellicer to whom the author is grateful for his constant aid and encouragement.

\section{On bitopological quasi-pseudometrization theorems}

In [3], Guthrie and Henry obtain a theorem of pseudometrization by using relatively complete families of functions. Here we introduce the concept of pairwise relatively complete family of functions which plays a similar role in bitopological quasi-pseudometrization.

2.1. Definition. Let $(X, \mathcal{P}, \mathcal{Q})$ be a bitopological space. Let $\mathscr{F}=\left\{f_{\alpha}: \alpha \in L\right\}$ a family of $\mathscr{P}$-1.s.c. and $\mathcal{Q}$-u.s.c. real-valued functions on $X$. Then:

(a) Family $\mathscr{F}$ is pairwise relatively complete if, for every $H \subset L, f(x)=\inf \left\{f_{\beta}(x)\right.$ : $\beta \in H\}$ is $\mathcal{P}$-1.s.c. and $F(x)=\sup \left\{f_{\beta}(x): \beta \in H\right\}$ is $\mathcal{L}$-u.s.c.

(b) Family $\mathscr{F}$ is pairwise semiequicontinuous at $x \in X$ if for each $\varepsilon>0$ there is a

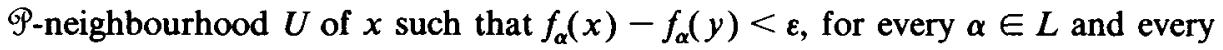
$y \in U$, and there is a 2 -neighbourhood $V$ of $x$ such that $f_{\alpha}(y)-f_{\alpha}(x)<\varepsilon$ for every $\alpha \in L$ and every $y \in V$.

(c) Family $\mathscr{F}$ is pairwise semiequicontinuous in $X$ if it is at every $x \in X$.

(d) Family $\mathscr{F}$ is punctually bounded in $X$ if for each $x \in X$, there is a real number $M>0$ such that $\left|f_{\alpha}(x)\right|<M$ for every $\alpha \in L$.

The proof of the next result is an adaptation of a technique of Guthrie and Henry [3].

2.2. Proposition. Let $(X, \mathscr{P}, \mathcal{Q})$ be a bitopological space and let $\mathscr{F}=\left\{f_{\alpha}\right.$ : $\alpha \in L\}$ be a family of real-valued functions on $X$. $\mathscr{F}$ is pairwise relatively complete if, and only if, it is pairwise semiequicontinuous and punctually bounded in $X$. 
Proof. Let us prove the 'if'. Let $x \in X$ and $H \subset L$. For each $\varepsilon>0$ there is a $\mathcal{P}$-neighbourhood $U$ of $x$ such that $f_{\beta}(x)-f_{\beta}(y)<\varepsilon / 2$, for every $y \in U$ and $\beta \in H$. Therefore $f=\inf \left\{f_{\beta}: \in H\right\}$ verifies $f(x)-f(y) \leqslant \varepsilon / 2$ and $f(x)-f(y)$ $<\varepsilon$ for every $y \in U$. Then $f$ is $\mathscr{P}$-1.s.c. in $X$. Similarly, $F=\sup \left\{f_{\beta}: \beta \in H\right\}$ is 2-u.s.c. in $X$.

Conversely, let $x \in X$ and $\varepsilon>0$. There is an $n \in \mathbf{N}$ such that $2^{-n}<\varepsilon$. For each $m \in \mathbf{Z}$, let

$$
L_{m n}=\left\{\alpha \in L:(m+1) 2^{-n-1} \leqslant f_{\alpha}(x) \leqslant(m+2) 2^{-n-1}\right\} .
$$

As $\inf \left\{f_{\alpha}(x): \alpha \in L\right\} \leqslant f_{\gamma}(x) \leqslant \sup \left\{f_{\alpha}(x): \alpha \in L\right\}$, we have $\mathscr{F}$ punctually bounded, and $L_{m n} \neq \varnothing$ only for a finite number of values $m$. Moreover $\cup_{m} L_{m n}$ $=L$ and when $L_{m n} \neq \varnothing$, by hypothesis, that

$$
\begin{array}{ll}
u_{m n}=\inf \left\{f_{\alpha}: \alpha \in L_{m n}\right\} & \text { is } \mathcal{P} \text {-1.s.c. in } X \text { and } \\
v_{m n}=\sup \left\{f_{\alpha}: \alpha \in L_{m n}\right\} & \text { is } 2 \text {-u.s.c. in } X ;
\end{array}
$$

therefore, for each $n \in N, U_{n}(x)=\cap_{m} u_{m n}^{-1}(] m 2^{-n-1},+\infty[)$ is an open $\mathscr{P}_{\text {. }}$ neighbourhood of $x$. If $y \in U_{n}(x)$ then $f_{\alpha}(y)>m 2^{-n-1}$ for every $\alpha \in L_{m n}$ and, as $f_{\alpha}(x) \leqslant(m+2) 2^{-n-1}$, we have $f_{\alpha}(x)-f_{\alpha}(y)<\varepsilon$ for every $\alpha \in L$. Similarly, $V_{n}(x)=\bigcap_{m} v_{m n}^{-1}(]-\infty,(m+3) 2^{-n-1}[)$ is an open 2-neighbourhood of $x$. If $y \in$ $V_{n}(x), f_{\alpha}(y)<(m+3) 2^{-n-1}$ and $f_{\alpha}(x) \geqslant(m+1) 2^{-n-1}$ for every $\alpha \in L_{m n}$; then, $f_{\alpha}(y)-f_{\alpha}(x)<\varepsilon$ for every $\alpha \in L$. Therefore $\mathscr{F}$ is pairwise semiequicontinuous.

2.3. Definition. $(X, \mathscr{P}, \mathcal{Q})$ is the pairwise initial bitopological space induced by the family $\mathscr{F}=\left\{f_{\alpha}: X \rightarrow \mathbf{R}, \alpha \in L\right\}$ if $\mathscr{P}$ is the initial topology of $\mathscr{F}$ respect to $\tau_{1}=\{\varnothing, \mathbf{R}] a,,+\infty[\forall a \in \mathbf{R}\}$ and $\mathcal{L}$ is the initial topology of $\mathscr{F}$ respect to $\tau_{2}=\{\varnothing, \mathbf{R}]-,\infty, a[\forall a \in \mathbf{R}\}$. We shall denote $\mathscr{P}=\tau_{1}(\mathscr{F})$ and $\mathcal{Q}=\tau_{2}(\mathscr{F})$.

2.4. TheOREM. The space $(X, \mathscr{P}, 2)$ is quasi-pseudometrizable if, and only if, it is the pairwise initial space induced by a $\sigma$-pairwise relatively complete family $\mathscr{F}$.

Proof. Let $d$ be a quasi-pseudometric compatible with $(X, \mathscr{P}, \mathcal{Q})$, that is such that $\mathcal{P}=\tau_{d}$ and $2=\tau^{d}$. It is not restriction to suppose $d \leqslant 1$. Let $\mathscr{F}=\left\{f_{p}: X \rightarrow \mathbf{R}\right.$ $\forall p \in X\}$ with $f_{p}(x)=1-d(p, x)$ for every $x \in X$. Let $\mathcal{G}=\left\{g_{p}: X \rightarrow \mathbf{R} \forall p \in\right.$ $X$ \} with $g_{p}(x)=d(x, p)-1$ for every $x \in X$. Let $x \in X$ and $\varepsilon>0$. For each element of the $\mathcal{P}$-open set $B_{d}(x, \varepsilon)$ we have $f_{p}(x)-f_{p}(y)=-d(p, x)+d(p, y)$ $\leqslant d(x, y)<\varepsilon$ for every $p \in X$. Therefore, $\mathscr{F}$ is pairwise semiequicontinuous and then $\mathscr{F}$ is pairwise relatively complete. In the same way we can prove that $\mathcal{G}$ is pairwise semiequicontinuous and pairwise relatively complete. Family $\mathscr{H}=\mathscr{F} \cup \mathcal{G}$ verifies $\tau_{1}(\mathcal{H}) \subset \mathscr{P}$ and $\tau_{2}(\mathscr{H}) \subset \mathcal{Q}$. Let $x \in X$ and let $U$ be a $\mathscr{P}$-neighbourhood 
of $x$; there is an $r_{1} \in \mathbf{R}$ with $0<r_{1}<1$ such that $B_{d}\left(x, r_{1}\right) \subset U$. Then $x \in\{y \in$ $\left.X: f_{x}(y)>1-r_{1}\right\} \subset B_{d}\left(x, r_{1}\right)$, that is, $\mathscr{P} \subset \tau_{1}(\mathcal{H})$. Similarly, we prove $\mathcal{L} \subset \tau_{2}(\mathcal{K})$ and, consequently, $(X, \mathcal{P}, \mathcal{Q})$ is the pairwise initial bitopological space induced by the pairwise relatively complete family $\mathscr{T C}$.

Conversely, let $\mathscr{F}=\bigcup_{n=1}^{\infty} \mathscr{F}_{n}$ with every $\mathscr{F}_{n}$ pairwise relatively complete. $\mathscr{P}=$ $\tau_{1}(\mathscr{F})$ and $\mathcal{L}=\tau_{2}(\mathscr{F})$. For every $n \in \mathbf{N}$ let the quasi-pseudometric

$$
d_{n}^{\prime}(x, y)=\sup \left\{\max (f(x)-f(y), 0): f \in \mathscr{F}_{n}\right\}
$$

for every $x, y$ in $X$. Let $d_{n}(x, y)=\min \left\{1, d_{n}^{\prime}(x, y)\right\}$ and

$$
d(x, y)=\sum_{n=1}^{\infty} 2^{-n} d_{n}(x, y) .
$$

Obviously $d$ is a quasi-pseudometric on $X$, and $0 \leqslant d \leqslant 1$., We shall prove that $\mathscr{P}=\tau_{d}$ and $\mathscr{Q}=\tau^{d}$. Let $x \in X$ and $h \in \mathscr{F}_{n}$ : for every $\varepsilon \in \mathbf{R}$ such that $0<\varepsilon<1$, let $\delta=\varepsilon 2^{-n}$ and $B_{d}(x, \delta) \in \tau_{d}$. If $d(x, y)<\delta$ then $d_{n}^{\prime}(x, y)=d_{n}(x, y)<\varepsilon$ and $h(x)-h(y) \leqslant \sup \left\{\max (f(x)-f(y), 0): f \in \mathscr{F}_{n}\right\}<\varepsilon$. Therefore $h$ is $\tau_{d}-1 . s . c$. and $\mathscr{P} \subset \tau_{d}$. Similarly, we prove that $h$ is $\tau^{d}$-u.s.c. and $\mathscr{Q} \subset \tau^{d}$.

In order to verify the opposite inclusions it is necessary to prove that $d_{n}^{\prime}$ is $\mathscr{P}$-u.s.c. with respect to the second co-ordinate and 2 -u.s.c. with respect to the first co-ordinate. Let $p \in X$ be fixed and $x \in X$; by Proposition 2.2, given $\varepsilon>0$ there is a $\mathscr{P}$-neighbourhood $U$ of $x$ with $f(x)-f(y)<\varepsilon / 2$ for every $f \in \mathscr{F}_{n}$ and $y \in U$. Then $d_{n}^{\prime}(x, y) \leqslant \varepsilon / 2<\varepsilon$. A similar argument proves that $d_{n}^{\prime}$ is 2-u.s.c. with respect to the first co-ordinate. Now we can deduce that $d$ is $\mathscr{P}$-u.s.c. with respect to the second co-ordinate. If we let $B_{d}(x, \varepsilon) \in \tau_{d}$, there is a $\mathscr{P}$-neighbourhood $U$ of $x$ such that $d(x, y)-d(x, x)<\varepsilon$ for every $y \in U$. Therefore $U \subset B_{d}(x, \varepsilon)$ and $\tau_{d} \subset \mathcal{P}$. Similarly we obtain $\tau^{d} \subset \mathcal{Q}$, and the theorem is proved.

Remark. If in Theorem 2.4 we put $\mathscr{P}=2$, we obtain [3] Theorem 4.

Salbany [11] gives the next definitions: let $(X, \mathscr{P}, 2)$ be a bitopological space and let $\mathcal{Q}=\left\{\left(A_{\alpha}, B_{\alpha}\right)\right\}$ be a set of pairs of subsets of $X$. $Q$ is $(\mathcal{Q}, \mathscr{P})$-locally finite if each $x \in X$ has a 2 -neighbourhood $V$ and $\mathscr{P}$-neighbourhood $U$ such that, except for finitely many $\left(A_{\alpha}, B_{\alpha}\right), U \cap B_{\alpha}=\varnothing$ or $V \cap A_{\alpha}=\varnothing . \mathscr{P}$ has an open pair base $\left\{\left(U_{\alpha}, V_{\alpha}\right)\right\}$ if for every $\alpha U_{\alpha}$ is $\mathscr{P}$-open, $V_{\alpha}$ is 2 -open, $U_{\alpha} \cup V_{\alpha}=X$, and for each $x \in U \in \mathscr{P}$, there is $\alpha$ such that $x \in X_{\alpha} \subset U_{\alpha} \subset U$.

2.5. CoROllaRY (Salbany). Let $(X, \mathcal{P}, \mathcal{2})$ be a pairwise normal space such that $\mathscr{P}$ has $a(\mathcal{Q}, \mathscr{P})-\sigma$-locally finite pair base and $\mathcal{Q}$ has a $(\mathscr{P}, \mathcal{Q})-\sigma-$ locally finite pair base, then $(X, \mathscr{P}, \mathcal{Q})$ is quasi-pseudometrizable. 
Proof. Let $\mathscr{Q}=\cup_{n=1}^{\infty} \mathbb{Q}_{n}$ a pair base of $\mathscr{P}$ where $\mathbb{Q}_{n}=\left\{\left(U_{\alpha}, V_{\alpha}\right)\right\}_{\alpha \in L}$ is $(\mathcal{Q}, \mathscr{P})$ locally finite for every $n$. As $(X, \mathcal{P}, \mathcal{Q})$ is pairwise normal, for each $\alpha$ there is an $f_{\alpha}: X \rightarrow[0,1] \mathscr{P}$-1.s.c. and 2 -u.s.c. function with $f_{\alpha}\left(X-U_{\alpha}\right)=\{0\}$ and $f_{\alpha}\left(X-V_{\alpha}\right)$ $=\{1\}$. Let $\mathscr{F}_{n}=\left\{f_{\alpha}:\left(U_{\alpha}, V_{\alpha}\right) \in \mathbb{Q}_{n}\right\}$. It is not difficult to prove that $\mathscr{F}_{n}$ is pairwise semiequicontinuous since $\mathscr{Q}_{n}$ is $(\mathcal{Q}, \mathscr{P})$-locally finite. Therefore $\mathscr{F}=\cup_{n=1}^{\infty} \mathscr{F}_{n}$ is a $\sigma$-pairwise relatively complete family. Obviously, $\tau_{1}(\mathscr{F}) \subset \mathscr{P}$ and $\tau_{2}(\mathscr{F}) \subset \mathcal{Q}$. Furthermore, if $x \in X$ and $U$ is a $\mathscr{P}$-neighbourhood of $x$, there is a pair $\left(U_{\alpha}, V_{\alpha}\right) \in \mathbb{Q}$ with $x \in X-V_{\alpha} \subset U_{\alpha} \subset U$, but $\left.\left.f_{\alpha}^{-1}(] 0,1\right]\right) \subset U_{\alpha}$ is a $\tau_{1}(\mathscr{F})$ neighbourhood of $x$, so $\mathscr{P} \subset \tau_{1}(\mathscr{F})$. In the same way one can obtain a $\sigma$-pairwise relatively complete family $\mathcal{G}=\cup_{n=1}^{\infty} \mathcal{G}_{n}$ so that $\tau_{1}(\mathcal{G}) \subset \mathscr{P}$ and $\tau_{2}(\mathcal{G})=\mathcal{2}$. Finally, $\mathcal{H}=\mathscr{F} \cup \mathcal{G}$ is a $\sigma$-pairwise relatively complete family with $\tau_{1}(\mathcal{K})=\mathscr{\rho}$ and $\tau_{2}(\mathcal{H})$ $=2$. Therefore, by Theorem $2.4,(X, \mathscr{P}, \mathcal{Q})$ is quasi-pseudometrizable.

Now we can obtain the bitopological version for the Nagata-Smirnov's theorem [11], Corollary 2.5. We shall obtain it as a simple corollary of next result, which also is proved in [10], but using a different technique.

2.6. CoROllary. Let $(X, \mathcal{P}, \mathcal{2})$ be a pairwise perfectly normal space such that $\mathscr{P}$

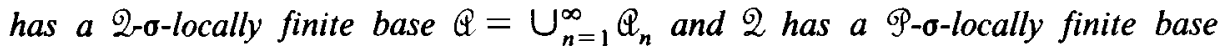
$\mathscr{B}=\cup_{n=1}^{\infty} \mathscr{B}_{n}$. Then, $(X, \mathscr{P}, \mathcal{Q})$ is quasi-pseudometrizable.

Proof. As $(X, \mathscr{P}, \mathcal{Q})$ is pairwise perfectly normal, for every $A \in \mathbb{Q}_{n}$ there is a $\mathscr{P}$-1.s.c. and 2 -u.s.c. function $f_{A, n}$ from $X$ into $[0,1]$, with $f_{A, n}^{-1}(0)=X-A$. Let $\mathscr{F}_{n}$ be the family of these functions, for every $n \in \mathbf{N} . \mathbb{Q}_{n}$ is 2 -locally finite: thus $\mathscr{F}_{n}$ is pairwise relatively complete. Therefore $\mathscr{F}=\cup_{n=1}^{\infty} \mathscr{F}_{n}$ is $\sigma$-pairwise relatively complete and, obviously, $\tau_{1}(\mathscr{F})=\mathscr{P}$ and $\tau_{2}(\mathscr{F}) \subset \mathcal{2}$. Similarly, we determine, from $\mathscr{B}$, a $\sigma$-pairwise relatively complete family $\mathcal{G}=\cup_{n=1}^{\infty} \mathcal{G}_{n}$ with $\tau_{1}(\mathcal{G}) \subset \mathcal{P}$ and $\tau_{2}(\mathcal{G})=$ 2. Finally, $\mathscr{H}=\mathscr{F} \cup \mathcal{G}$ is a $\sigma$-pairwise relatively complete family with $\tau_{1}(\mathcal{K})=\mathscr{P}$ and $\tau_{2}(\mathcal{K})=2$. Then, following Theorem $2.4,(X, \mathscr{P}, \mathcal{Q})$ is quasi-pseudometrizable.

2.7. Corollary (Salbany). Let ( $X, \mathcal{P}, \mathcal{2})$ be a pairwise regular space. If $P$ has a

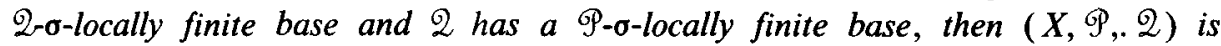
quasi-pseudometrizable.

Proof. Taking into account [7], Theorem $3.5,(X, \mathscr{P}, \mathcal{Q})$ is pairwise perfectly normal space and, by Corollary 2.6 , it is quasi-pseudometrizable. 
Reilly [8] gives next definitions: if $D$ is a family of quasi-pseudometrics on the set $X$, we say that $D$ is a quasi-gauge. The topology $\tau(D)$ having as a subbase the family $\mathscr{B}(D)$ of all balls $B_{d}(x, d, \varepsilon)$ with $d \in D, x \in X$ and $\varepsilon>0$ is called the topology induced on $X$ by the quasi-gauge $D$. If $D$ is a quasi-gauge, then the family $E=\{e: e$ is conjugate to some $d \in D\}$ is the conjugate quasi-gauge of $D$. A quasi-gauge structure for a bitopological space $(X, \mathcal{P}, \mathcal{Q})$ is a pair $(D, E)$ of conjugate quasi-gauges on $X$ such that $\mathscr{P}=\tau(D)$ and $\mathcal{L}=\tau(E)$.

2.8. Theorem. $A$ bitopological space $(X, \mathscr{P}, \mathcal{Q})$ is quasi-pseudometrizable if, and only if, it has a countable quasi-gauge structure.

Proof. Let $(D, E)$ be a countable quasi-gauge structure for $(X, 9,2)$. Let $D=\left\{d_{n}\right\}_{n=1}^{\infty}$ and $E=\left\{e_{n}\right\}_{n=1}^{\infty}, e_{n}$ being the conjugate of $d_{n}$. It is not a restriction to suppose $0 \leqslant d_{n} \leqslant 1$. For every $n \in \mathbf{N}$ and every $x \in X$, let $f_{x, n}(y)=1-$ $d_{n}(x, y)$; let $\mathscr{F}_{n}=\left\{f_{x, n}: x \in X\right\}$. It is easy to prove that $\mathscr{F}_{n}$ is pairwise semiequicontinuous and, therefore, $\mathscr{F}=\cup_{n=1}^{\infty} \mathscr{F}_{n}$ is $\sigma$-pairwise relatively complete. For every $n \in \mathrm{N}$ and $x \in X$, let $g_{x, n}(y)=e_{n}(x, y)-1$; let $\mathcal{G}_{n}=\left\{g_{x, n}: x \in X\right\}$, then $\mathcal{G}=\cup_{n=1}^{\infty} \mathcal{G}_{n}$ is $\sigma$-pairwise relatively complete. The family $\mathcal{H}=\mathscr{F} \cup \mathcal{G}$ is $\sigma$-pairwise relatively complete, $\tau_{1}(\mathcal{K})=\mathscr{P}$ and $\tau_{2}(\mathcal{H})=2$. Then, $(X, \mathscr{P}, \mathcal{2})$ is quasipseudometrizable. The converse is obvious.

In [1], Theorem 4 , it is proved that if $\left(X, \mathscr{Q}, \mathcal{Q}^{-1}\right)$ is a quasi-uniform space, and $\mathcal{U}$ has a countable base, then $\left(X, \tau_{\mathcal{Q}}, \tau_{\mathcal{Q}^{-1}}\right)$ is quasi-pseudometrizable. By using this result, we can give an easy proof of next theorem:

2.9. ThEOREM (Pareek). ( $X, \mathscr{P}, \mathcal{Q})$ is quasi-pseudometrizable if, and only if, there are functions $\mathbf{g}: \mathbf{N} \times X \rightarrow P$ and $h: \mathbf{N} \times X \rightarrow \mathcal{Q}$ such that:

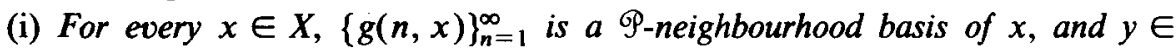
$g(n, x)$ implies $g(n, y) \subset g(n-1, x)$.

(ii) For every $x \in X,\{h(n, x)\}_{n=1}^{\infty}$ is a 2 -neighbourhood basis of $x$, and $y \in$ $h(n, x)$ implies $h(n, y) \subset h(n-1, x)$.

(iii) $y \in g(n, x)$ if, and only if, $x \in h(n, y)$.

Proof. If (i), (ii) and (iii) are verified, we can define, as in [12], Theorem 2.1 $U_{n}=\cup_{x \in X}\{x\} \times g(n, x)$ for each $n \in \mathbf{N}$. Then, if $U_{0}=X \times X,\left\{U_{n}\right\}_{n=0}^{\infty}$ is a base of a quasi-uniformity $\mathcal{Q}$ on $X$ with $\tau_{\mathfrak{Q}}=\mathscr{P}$.

For every $x \in X, U_{n}^{-1}[x]=\left\{y \in X:(y, x) \in U_{n}\right\}=\{y \in X: x \in g(n, y)\}=$ $\{y \in X: y \in h(n, x)\}=h(n, x)$, therefore $\tau_{\mathscr{Q}^{-1}}=2$. Then, by [1], Theorem 4, $(X, \mathscr{P}, \mathcal{Q})$ is quasi-pseudometrizable. The converse is obvious. 


\section{References}

[1] P. Fletcher, H. B. Hoyle III and C. W. Patty, 'The comparison of topologies', Duke Math. J. 36 (1969), 325-331.

[2] J. A. Guthrie and M. Henry, 'Metrization, paracompactness and real-valued functions', Fund. Math. 95 (1977), 49-53.

[3] J. A. Guthrie and M. Henry, 'Metrization, paracompactness and real-valued functions II', Fund. Math. 96 (1979), 13-20.

[4] J. C. Kelly, 'Bitopological spaces', Proc. London Math. Soc. 13 (1963), 71-89.

[5] E. P. Lane, 'Bitopological spaces and quasi-uniform spaces', Proc. London Math. Soc. 17 (1967), 241-256.

[6] C. M. Pareek, 'Bitopological spaces and quasi-metric spaces', J. Univ. Kuwait Sci. 6 (1980), 1-7.

[7] C. W. Patty, 'Bitopological spaces', Duke Math. J. 34 (1967), 387-392.

[8] I. L. Reilly, 'Quasi-gauge spaces', J. London Math. Soc. 6 (1973), 481-487.

[9] H. Ribeiro, 'Sur les éspaces á métrique faible', Portugal. Math. 4 (1943), 21-40.

[10] S. Romaguera, 'On bitopological quasi-pseudometrization', J. Austral. Math. Soc., to appear.

[11] S. Salbany, 'Quasi-metrization of bitopological spaces', Arch. Math. 23 (1972), 299-306.

[12] R. A. Stoltenberg, 'On quasi-metric spaces', Duke Math. J. 36 (1969), 65-72.

Departmento de Matemáticas II

ETSICCP

Universidad Politécnica

Valencia-22

Spain 